Europhysics Letters

PREPRINT

\title{
Mesoscopic Kondo effect of a quantum dot embedded in an Aharonov-Bohm ring with intradot spin-flip scattering
}

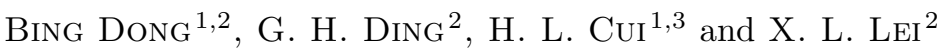 \\ 1 Department of Physics and Engineering Physics, Stevens Institute of Technology, \\ Hoboken, New Jersey 07030, USA \\ 2 Department of Physics, Shanghai Jiaotong University, 1954 Huashan Road, Shanghai \\ 200030, China \\ 3 School of Optoelectronics Information Science and Technology, Yantai University, \\ Yantai, Shandong, China
}

PACS. 72.15.Qm - Scattering mechanisms and Kondo effect.

PACS. 73.23. Ra - Persistent currents.

PACS. 73.23.Hk - Coulomb blockade; single-electron tunneling.

\begin{abstract}
.
We study the Kondo effect in a quantum dot embedded in a mesoscopic ring taking into account intradot spin-flip scattering $R$. Based on the finite- $U$ slave-boson mean-field approach, we find that the Kondo peak in the density of states is split into two peaks by this coherent spin-flip transition, which is responsible for some interesting features of the Kondo-assisted persistent current circulating the ring: (1) strong suppression and crossover to a sine function form with increasing $R$; (2) appearance of a "hump" in the $R$-dependent behavior for odd parity. $R$-induced reverse of the persistent current direction is also observed for odd parity.
\end{abstract}

Intensive attention has been paid to the Kondo effect in a quantum dot (QD), [1,2] in which formation of a bound state between the local spin and conduction electrons induces a Kondo peak in the density of states (DOS) at the Fermi level $E_{\mathrm{F}}$, consequently leading to strong enhancement of the linear conductance ultimately to the ideal value $2 e^{2} / h$ below the bulk Kondo temperature $T_{\mathrm{K}}^{0}$. Associated with this energy scale, the characteristic length of the bound state wave-function, the so-called Kondo screening cloud defined as $\xi_{\mathrm{K}}^{0} \approx \hbar v_{\mathrm{F}} / T_{\mathrm{K}}^{0}$ $\left(v_{\mathrm{F}}\right.$ is the Fermi velocity), is itself mesoscopic size (of the order of $1 \mu \mathrm{m}$ ).

The easy control of major parameters of these artificial atoms in a wide range makes it possible to study the Kondo effect even when the size of the lead is comparable to the size of screening cloud $\xi_{\mathrm{K}}^{0}$. Recently, Affleck and Simon [3] suggested that the setup of a QD embedded in a closed mesoscopic Aharonov-Bohm (AB) ring is an ideal tool to detect this cloud because their perturbation calculations found that the persistent current (PC) in such a geometry is a universal scaling function of $\xi_{\mathrm{K}}^{0} / L$ ( $L$ is the ring circumference), and crosses over from the saw-tooth of perfect ring in the limit $\xi_{\mathrm{K}}^{0} / L \ll 1$ to a sine function with vanishing magnitude at $\xi_{\mathrm{K}}^{0} / L \gg 1$. Besides, slave-boson mean-field (SBMF) evaluation disclosed that this finite size effect affects drastically the Kondo resonance in a way that depending on the

(C) EDP Sciences 
total number of electrons (modelo 4). [4,5] There was also a variational calculation about this problem. [6]

On the other hand, the recent issue has been focused on what happens to the Kondo physics if an intrinsic spin-flip scattering is stirred in the QD and/or even when the conduction band is allowed itself to be ferromagnetic metal. [9-15] By means of the SBMF approach, Ma et al. [13] and López et al. [14] found respectively a splitting of the zero-bias peak of the nonlinear differential conductance when the spin-flip scattering amplitude $R$ is of the order of the Kondo temperature, indicating the Kondo peak splitting and suppression in the DOS. This has been further confirmed by using an exact theoretical method, numerical renormalization group technique. [15]

Actually, the electron in the semiconductor QD suffers inevitably intrinsic relaxations (decoherence) due to the spin-orbital interaction [7] or the hyperfine-mediated spin-flip transition, [8] which can be modeled by a phenomenological spin-flip scattering term $R$ as done in these previous papers. [13-15] Naturally, it would be interesting to study the modification of the Kondo resonance due to the intradot spin-flip transition at a mesoscopic situation $\xi_{\mathrm{K}}^{0} / L \sim 1$ and its effect on the PC.

In this letter, based on the finite- $U$ SBMF approach initially developed by Kotliar and Ruckenstein, [16] we perform a detailed analysis for this issue. With the help of four slave boson parameters, this SBMF approach not only allows to deal with finite on-site Coulomb interaction but also is expected to give good results both for the charge fluctuation and spin fluctuation of the Anderson impurity model, which enables us to thoroughly analyze this problem (the Kondo-type DOS in QD without attaching to leads and the PC circulating the ring) sweeping across the whole regions, i.e., Kondo, mixed-valence, and empty-orbital regimes. $[5,13]$

We consider a $\mathrm{QD}$ with a single bare level $\epsilon_{d}$ having a finite on-site Coulomb repulsion $U$ and an intradot spin-flip scattering $R$ is embedded in a mesoscopic AB ring, modeled by a one-dimensional tight-binding Hamiltonian with the nearest neighbor hopping $t$, through tunneling $t_{\eta}(\eta=L, R)$. According to the finite- $U$ slave-boson approach, [16] we introduce four auxiliary boson operators $e, p_{\sigma}$, and $d$, which are associated respectively with the empty, singly occupied and doubly occupied electron states at the QD, to describe the above physical problem without correlation terms in an enlarged space with constraints. In terms of the auxiliary particle representation, the effective Hamiltonian for this system (totally $N$ sites including the QD) can be written as:

$$
\begin{aligned}
H= & -t \sum_{j=1}^{N-2} \sum_{\sigma}\left(c_{j \sigma}^{\dagger} c_{j+1 \sigma}+\text { H.c. }\right)+\sum_{\sigma} \epsilon_{d} c_{d \sigma}^{\dagger} c_{d \sigma} \\
& +U d^{\dagger} d+R c_{d \uparrow}^{\dagger} c_{d \downarrow}+R c_{d \downarrow}^{\dagger} c_{d \uparrow}-\sum_{\sigma}\left(t_{L} z_{\sigma}^{\dagger} c_{d \sigma}^{\dagger} c_{1 \sigma}+t_{R} e^{i \phi} c_{N-1 \sigma}^{\dagger} c_{d \sigma} z_{\sigma}+\text { H.c. }\right) \\
& +\lambda^{(1)}\left(\sum_{\sigma} p_{\sigma}^{\dagger} p_{\sigma}+e^{\dagger} e+d^{\dagger} d-1\right)+\sum_{\sigma} \lambda_{\sigma}^{(2)}\left(c_{d \sigma}^{\dagger} c_{d \sigma}-p_{\sigma}^{\dagger} p_{\sigma}-d^{\dagger} d\right),
\end{aligned}
$$

where $c_{d \sigma}^{\dagger}\left(c_{d \sigma}\right)$ is the creation (annihilation) operators of electrons in the QD, the three Lagrange multipliers $\lambda^{(1)}$ and $\lambda_{\sigma}^{(2)}$ impose the constraints, and in the hopping term the fermion operator $c_{d \sigma}$ is replaced by $c_{d \sigma} z_{\sigma}$ with a many-body correction factor $z_{\sigma}=(1-$ $\left.d^{\dagger} d-p_{\sigma}^{\dagger} p_{\sigma}\right)^{-\frac{1}{2}}\left(e^{\dagger} p_{\sigma}+p_{\bar{\sigma}}^{\dagger} d\right)\left(1-e^{\dagger} e-p_{\bar{\sigma}}^{\dagger} p_{\bar{\sigma}}\right)^{-\frac{1}{2}}$. The phase factor $\phi$ is defined by $2 \pi \Phi / \Phi_{0}$, in which $\Phi$ and $\Phi_{0}=h / e$ are the external magnetic flux enclosed by the ring and the flux quantum, respectively. The coupling strength $\Gamma$ between the $\mathrm{QD}$ and the ring is associated with the ring DOS $\rho\left(E_{\mathrm{F}}\right)$ and hopping $t\left(E_{\mathrm{F}}\right)$ at the Fermi energy as $\pi \rho\left(E_{\mathrm{F}}\right)\left|t\left(E_{\mathrm{F}}\right)\right|^{2}$. Here 
we would like to point out that the intrinsic spin-flip processes lift the level degeneracy of the QD, yielding $\epsilon_{d} \pm R$. Because of no special spin direction, it seems that our model is equivalent to the one of the QD under external magnetic field (Zeeman effect). However, we still prefer the present form since it provides a direct description of spin relaxation in the QD and can give us a heuristic comprehension of the spin-relaxation effects on the PC, although the spin is flipped without any type of dissipation in this simple model. Moreover, our results obtained in the following are also applicable for the case of applying external magnetic field. We consider the half-filled case $\left(N_{e}=N\right)$ in this letter, and in the continuum limit we have $E_{\mathrm{F}}=0, \rho(0)=N /(2 \pi t),|t(0)|^{2}=2\left(t_{L}^{2}+t_{R}^{2}\right) / N$, and $\Gamma_{\eta}=t_{\eta}^{2} / t(\eta=L, R)$.

At zero temperature, we utilize the mean-field approximation in which all the boson operators are replaced by real $c$ numbers (variational parameters). Consequently we can solve the noninteracting Hamiltonian Eq. (1) exactly by numerical diagonalization and evaluate the expectation value of any operators in the ground state $|0\rangle$ by summing over consecutive eigenlevels up to the highest occupied single particle energy level (HOEL). Minimizing the ground state energy $E_{\mathrm{g}}$ [expectation value of the Hamiltonian Eq. (11) in the ground state] with respect to these variational parameters together with the three constraints form the basic self-consistent equations to determine these unknown parameters within the mean-field scheme. [5] Once solving the set of nonlinear equations, one can calculate the electron DOS $\rho_{\sigma}(\omega)$ for the QD by

$$
\rho_{\sigma}(\omega)=-\frac{1}{\pi}\left|z_{\sigma}\right|^{2} \operatorname{Im}\left\langle v\left|c_{d \sigma}\left(\omega+i 0^{+}-H\right)^{-1} c_{d \sigma}^{\dagger}\right| v\right\rangle
$$

( $|v\rangle$ stands for the vaccum state) and the PC $I$ by

$$
I=-\frac{e}{\hbar} \frac{\partial E_{\mathrm{g}}}{\partial \phi}=\frac{i e}{\hbar} \sum_{\sigma}\langle 0| t_{R} z_{\sigma} e^{i \phi} c_{N-1 \sigma}^{\dagger} c_{d \sigma}-\text { H.c. }|0\rangle .
$$

The mesoscopic "Kondo correlation energy" $T_{\mathrm{K}}$ is defined as the binding energy between the $\mathrm{QD}$ and the ring:

$$
T_{\mathrm{K}}=\epsilon_{d}-\epsilon_{\mathrm{F}}-\left(E_{\mathrm{g}}-E_{\mathrm{g}}^{0}\right),
$$

in which $\epsilon_{\mathrm{F}}$ is the energy of the HOEL corresponding to the Fermi energy and $E_{\mathrm{g}}^{0}$ is the ground state energy of the $N-1$ sites tight-binding Hamiltonian with the same electron number $N_{e}$.

In the following, we present our calculations only for the mesoscopic ring with $\xi_{\mathrm{K}}^{0} / L \sim 1$ $(L=N)$ in the particle-hole symmetric region. We will also take $t=1, t_{L}=t_{R}=0.35, \Gamma=$ $\Gamma_{L}+\Gamma_{R}=0.245$ and choose the on-site interaction $U=8 \Gamma$, for the symmetric (asymmetric) case $\epsilon_{d}=-U / 2(-U / 4)$ corresponding to $T_{\mathrm{K}}^{0}=0.013(0.034)$ or $\xi_{\mathrm{K}}^{0}=148(59)$.

Figure 1 shows the dot DOS $\rho_{\sigma}(\omega)$ in the symmetric case $\epsilon_{d}=-U / 2$ for different values of the intradot spin-flip scattering $R$ in terms of the Kondo temperature $T_{\mathrm{K}}^{0}=U \sqrt{\beta} \exp (-\pi / \beta) / 2 \pi$ $\left[\beta=-2 U \Gamma / \epsilon_{d}\left(U+\epsilon_{d}\right)\right]$. It is obvious that in the mesoscopic case $L \sim \xi_{\mathrm{K}}^{0}$, the bulk single Kondo peak becomes a set of subpeaks depending on the total numbers of lattice sites $N$ (modulo 4), which are roughly attributed to the patterns of the HOEL and lowest unoccupied energy level (LUEL) of the Hamiltonian Eq. (11). [4] For $N=4 n$, the energies of the HOEL $\left(\epsilon_{\mathrm{F}}\right)$ and the LUEL are both nearly equal to zero, so there is a single main peak in DOS pinned at $\omega=0$ together with several small peaks located at other single particle energy levels. For $N=4 n+2, \epsilon_{\mathrm{F}}$ is negative with a distinguishable value and LUEL is equal to $-\epsilon_{\mathrm{F}}$. As a result, two Kondo peaks appear around $\omega=0$ with the same amplitudes. While in the cases of odd parity $(N=4 n \pm 1), \epsilon_{\mathrm{F}}$ is nearly below or above zero, respectively, leading to a single Kondo resonance slightly shifted from the Fermi energy. This shifting of the Kondo peak from 


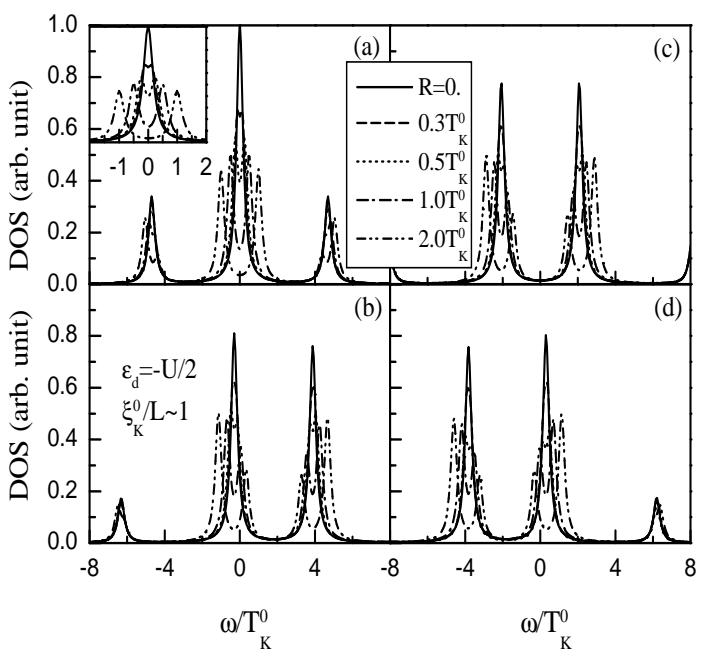

Fig. 1

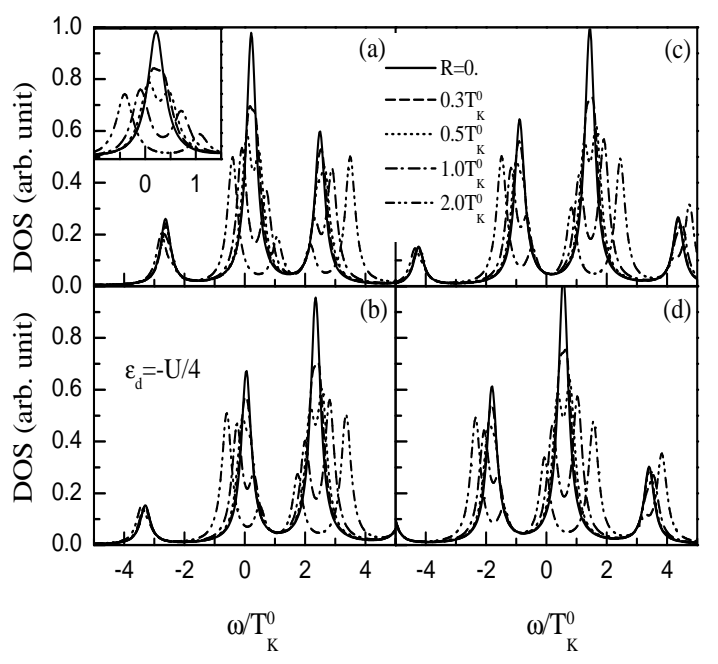

Fig. 2

Fig. 1 - The dot DOS for the symmetric case $\epsilon_{d}=-U / 2$ at various spin-flip processes $R$ without magnetic flux. The number of the lattice sites $N$ are (a) $4 n$, (b) $4 n+1$, (c) $4 n+2$, and (d) $4 n+3$. Inset: $\rho_{\sigma}(\omega)$ around $\omega=0$ at $N=4 n$.

Fig. 2 - The dot DOS $\rho(\omega)$ for the asymmetric case $\epsilon_{d}=-U / 4$. The other parameters are the same as in Fig. 1.

the Fermi energy $\epsilon_{\mathrm{F}}$ in the odd parity will cause the reduction of PC magnitude (see Fig. 4 below). On the other hand, introduction of the spin-flip scattering $R$ always reduces and splits every Kondo peak regardless of parity. Moreover, with increasing $R$ the DOS suppresses in a more pronounced way, and the two split Kondo peaks are entirely symmetric for $N=4 n$. However, the situation is somewhat different for the asymmetric system $\epsilon_{d}=-U / 4$ as shown in Fig. 2. The profiles of DOS wholly move a little bit towards the high energy. This is because the asymmetric system allows charge fluctuations to certain extent and consequently increases the resulting single particle energy levels delicately. Associated with the spin-flip induced splitting, this feature results in a novel $R$-dependent behavior of the PC (see below).

We also study the splitting width $\delta$ of the main Kondo peak at $N=4 n$ and plotted it as a function of $R$ in Fig. 3(a), which shows a significant reduction of the width $\delta$ in contrast to the predicted value $2 R$ by measuring nonlinear differential conductance through a QD connected to macroscopic leads. [14] Actually, the exact Bethe-ansatz solution for the QD also reveal explicitly this large suppression of the peak splitting due to the strong correlations in the presence of the external Zeeman field. [17] Besides, Fig. 3(b) displays that, for the fixed $R$, the peak splitting shrinks when the QD departs away from the particle-hole symmetric point. In Fig. 3(c) and Fig. 3(d), we summarize the calculated mesoscopic Kondo temperatures $T_{\mathrm{K}}$ with variation of $R$ by using Eq. (4). We find that: (1) The finite size effect remarkably increases the binding energy between the QD and the ring, so the mesoscopic Kondo temperatures $T_{\mathrm{K}}$ are much higher than the corresponding bulk values $T_{\mathrm{K}}^{0}$; (2) Charge fluctuations can decrease this binding energy (because it increases the single particle energy levels), and accordingly, lead to a smaller $T_{\mathrm{K}}$ in the particle-hole asymmetric region; (3) The behaviors of $T_{\mathrm{K}}$ with rising of $R$ depend on the parity: For even parity $N=4 n \pm 2, T_{\mathrm{K}}$ decreases first with small amplitude and then begins to increase, while in the case of odd parity $N=4 n \pm 1, T_{\mathrm{K}}$ increases 

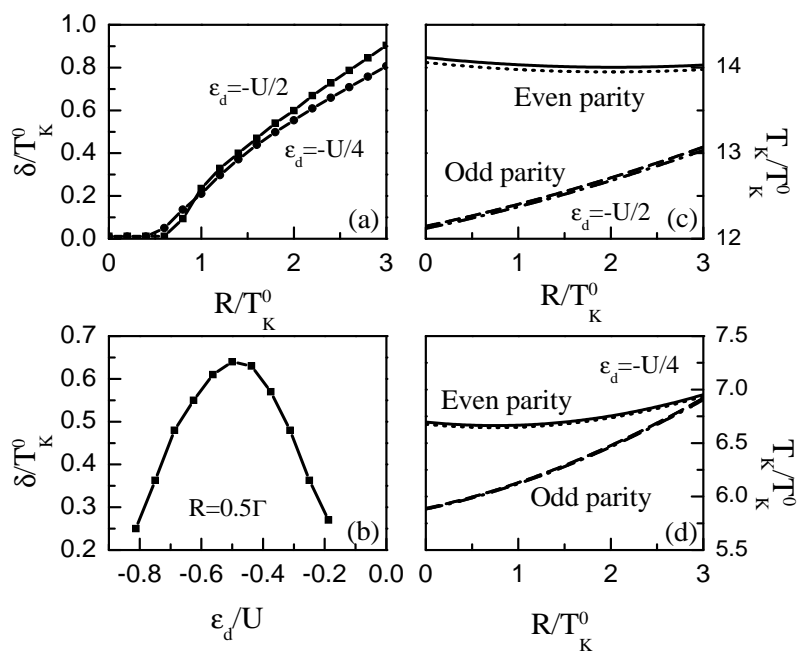

Fig. 3 - (a) Splitting $\delta$ of the main Kondo peak vs. $R$ for $\epsilon_{d}=-U / 2$ and $-U / 4$ at $N=4 n$ and zero magnetic flux. (b) $\delta$ as a function of $\epsilon_{d}$ at $R=0.5 \Gamma$. Kondo temperature $T_{\mathrm{K}} / T_{\mathrm{K}}^{0}$ vs. $R$ for (c) the symmetric and (d) the asymmetric systems at mesoscopic condition $L \sim \xi_{\mathrm{K}}^{0}$ and $\phi=\pi / 2$.

monotonously, implying that the Kondo screening cloud shrinks due to the spin-flip scattering.

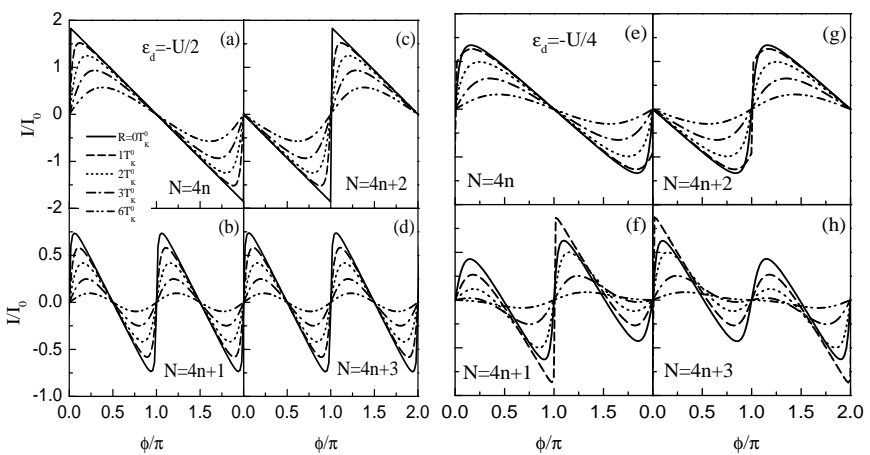

Fig. 4 - Normalized PCs vs. magnetic flux at various $R$ for $\epsilon_{d}=-U / 2(\mathrm{a})-(\mathrm{d})$ and $\epsilon_{d}=-U / 4(\mathrm{e})-(\mathrm{h})$. $I_{0}=2 t / N$ is the corresponding $\mathrm{PC}$ of an ideal $N$ sites ring.

Now we turn to calculation of the PC circulating around the ring, which is believed to be a useful way to detect the Kondo resonance due to the fact that the PC is closely related to electron transmission through the QD embedded in the ring. We illustrate in Fig. 4 the $R$ dependent PC $I / I_{0}$ vs. magnetic flux at mesoscopic condition for $\epsilon_{d}=-U / 2$ and $\epsilon_{d}=-U / 4$. In the absence of spin-flip transition, (1) the PC has an analogous structure of an ideal ring, implying high transmission through the QD; (2) its direction depends on the parity of the sites number $N$; (3) the magnitude of the PC for the odd parity is much smaller than that for the even parity because of the Kondo peak slightly shifting in the case of the odd parity; and (4) the perfect symmetric structures for the particle-hole symmetric point $\epsilon_{d}=-U / 2$ guarantee $I^{4 n+1}(\phi)=I^{4 n-1}(\phi)$, but there are no this feature for the asymmetric systems. As expected, applying spin-flip scattering generally lowers the magnitude of the PC due to weakening of 


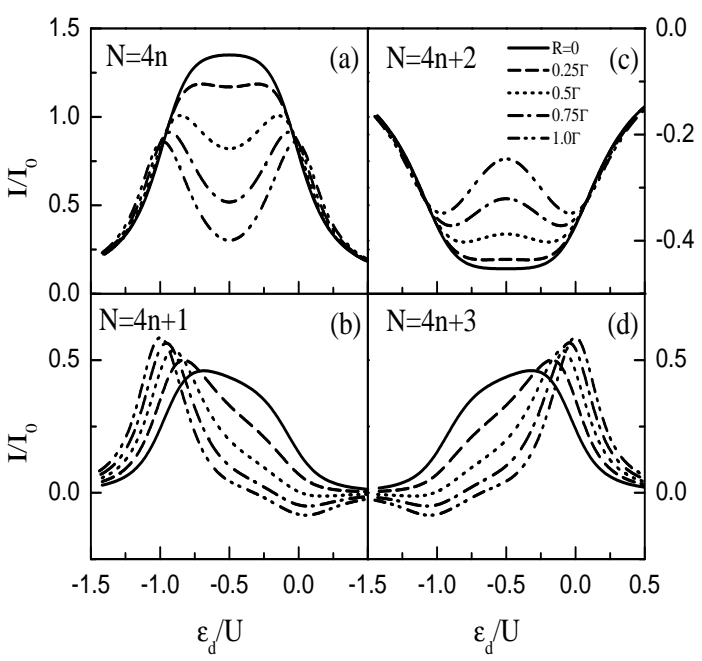

Fig. 5

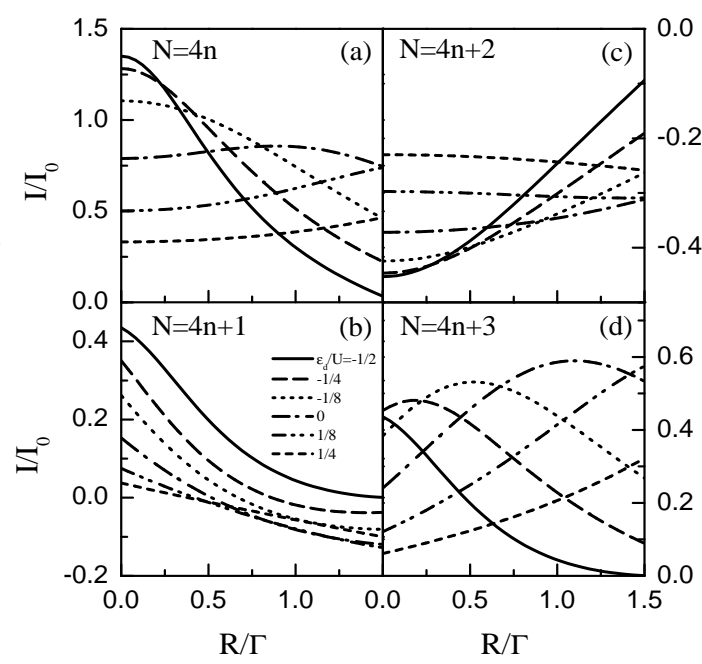

Fig. 6

Fig. 5 - PC vs. energy level $\epsilon_{d} / U$ of the QD for different parities and several spin-flip transitions at $\phi=\pi / 4$.

Fig. 6 - The PC as a function of spin-flip scattering $R$ at different energy levels of the QD.

the Kondo resonance and varies its profile gradually to a rough sinusoidal, even though the screening cloud is also reduced at the same time. More strikingly, strong spin-flip scattering even changes the sign of $d I^{4 n+1}(\phi) / d \phi$ at small $\phi$ for the asymmetric system from positive to negative, indicating that the symmetry of the HOEL changes and the system becomes diamagnetic.

Figure 5 displays the $\mathrm{PC}$ as a function of the QD level. For even parities, the PC exhibits symmetric feature around the central point $\epsilon_{d}=-U / 2$ and unique magnetic response properties (being paramagnetic for $N=4 n$, while diamagnetic for $N=4 n+2$ ) in the whole range of energy levels. In the region where the Kondo effect plays a dominating role, the PC is obviously enhanced in contrast to its corresponding ideal value $\left(I / I_{0}>1\right)$. However, significant suppression of the Kondo resonance due to increasing $R$ can reduce the Kondo-assisted $\mathrm{PC}$ rapidly. But in both non-Kondo and deep Kondo regimes, we observe this reduction gets gradually weak and even a slight increase because of charge fluctuations. Finally at a large scattering $R=1.0 \Gamma$, the $\mathrm{PC}$ shows a Coulomb blockade pattern in analogy with the behavior of linear conductance through QD over the Kondo temperature. For the case of odd parity, the situation is quite different. The Kondo-assisted PC is asymmetric and a remarkable feature can be clearly seen $I^{4 n+1}\left(\epsilon_{d} / U\right)=I^{4 n+3}\left(\left|\epsilon_{d} / U\right|-1\right)$ due to the symmetry properties of DOS in the finite- $U$ Anderson model. Two more striking results can be observed (we only address for $N=4 n+3$ here): (1) The PC is a monotonous function of the spin-flip $R$ at $\epsilon_{d}>0$ and $\epsilon_{d}<-U / 2$, whereas there is no universal $R$ behavior in the middle widow; (2) As mentioned above, the PC may change direction with increase of $R$ for $\epsilon_{d}<-U / 2$. We summarize these $R$-dependent features of the PC more clearly in Fig. 6 , in which we plot the $\mathrm{PC}$ as a function of $R$. Explicitly in Fig. 6(d), the PC exhibits a "hump" structure for $\epsilon_{d} / U=-\frac{1}{4}$ and $-\frac{1}{8}$ as increasing $R$, which is due to the fact that the Kondo resonance is shifted away from the Fermi energy as shown in Fig. 2(d).

In short, we have investigated the Kondo resonance in a QD embedded in a mesoscopic 
Bing Dong, G. H. Ding, H. L. Cui and X. L. Lei: Mesoscopic Kondo effect of A QuAntum Dot Embedded in An Aharonor

AB ring taking into account intradot spin-flip scattering $R$ or application of magnetic field in the QD by means of the finite- $U$ SBMF approach. The Kondo resonance splits into two clear peaks (Zeeman effect) with a largely reduced width when increasing $R$. We also reported the $R$-dependent PC behaviors in both Kondo and non-Kondo regimes, which can measure this Kondo effect in an equilibrium condition. This model is experimentally accessible for the update techniques and provides a possible test for the influence of spin-flip transition on the Kondo screening.

$$
* * *
$$

BD and HLC is supported by the DURINT Program administered by the US Army Research Office. XLL is supported by Major Projects of National Natural Science Foundation of China, the Special Founds for Major State Basic Research Project and the Shanghai Municipal Commission of Science and Technology.

\section{REFERENCES}

[1] Goldhaber-Gordon D., ET AL., Nature (London), 391 (1998) 156.

[2] Cronenwett S.M., et AL., Science, 281 (1998) 540.

[3] Affleck I. and Simon P., Phys. Rev. Lett., 86 (2001) 2854.

[4] Hu H., Zhang G.M., and Yu L., Phys. Rev. Lett., 86 (2001) 5558.

[5] Ding G.H. and Dong B., Phys. Rev. B, 67 (2003) 195327.

[6] Kang K. and Shin S.C., Phys. Rev. Lett., 85 (2000) 5619.

[7] Mozyrsky D., Fedichkin L., Gurvitz S.A., and Berman G.P., Phys. Rev. B, 66 (2002) 161313.

[8] Erlingsson S.I., Nazarov Yu.V., Phys. Rev. B, 66 (2002) 155327.

[9] Rudziński W. and Barnaś J., Phys. Rev. B, 64 (2001) 085318.

[10] Souza F.M., ET AL., cond-mat/0209263, (2002) .

[11] Zhang P., Xue Q.K., Wang Y.P., and Xie X.C., Phys. Rev. Lett., 89 (2002) 286803.

[12] Dong B., Cui H.L., Liu S.Y., and Lei X.L., J. Phys.: Condens. Matter, 15 (2003) 8435.

[13] MA J. and LEI X.L., cond-mat/0309520, (2003).

[14] López R. and SÁnchez D., Phys. Rev. Lett., 90 (2003) 116602.

[15] Choi M.-S., SÁnchez D., and López R., cond-mat/0305107, (2003) .

[16] Kotliar G., and Ruckenstein A.E., Phys. Rev. Lett., 57 (1986) 1362.

[17] Moore J.E., and Wen X.G., Phys. Rev. Lett., 85 (2000) 1722. 